\title{
Current-Dependent Exchange-Correlation Potential for Dynamical Linear Response Theory
}

\author{
G. Vignale \\ Department of Physics, University of Missouri, Columbia, Missouri 65211 \\ Walter Kohn \\ Department of Physics, University of California, Santa Barbara, California 93106-4030
}

(Received 26 April 1996)

\begin{abstract}
The frequency-dependent exchange-correlation potential, which appears in the usual Kohn-Sham formulation of a time-dependent linear response problem, is a strongly nonlocal functional of the density, so that a consistent local density approximation generally does not exist. This problem can be avoided by choosing the current density as the basic variable in a generalized Kohn-Sham theory. This theory admits a local approximation which, for fixed frequency, is exact in the limit of slowly varying densities and perturbing potentials. [S0031-9007(96)01133-7]

PACS numbers: 71.45.Gm, 73.20.Dx, 73.20.Mf, 78.30.Fs
\end{abstract}

The time-dependent density functional theory (TDFT) of Runge and Gross [1] potentially holds great promise as a tool for studying the dynamics of many-particle systems, as well as for the computation of excitation energies which are not accessible within the ordinary static density functional theory. At sufficiently low frequencies applications of the so-called adiabatic local density approximation (ALDA) [2] have given very useful results. Progress in the application of this theory to higher frequency phenomena has been hindered by inconsistencies in the local density approximation for the frequency dependent exchange-correlation (xc) potential [3,4]. This paper presents a resolution of these difficulties, by providing the correct form of the frequency dependent xc potential in the regime of linear response and slowly varying densities and perturbing potentials.

Our objective is the determination of the linear density response $n_{1}(\vec{r}, \omega) e^{-i \omega t}$ of a system of interacting electrons in their ground state to a time-dependent potential $v_{1}(\vec{r}, \omega) e^{-i \omega t}$. In TDFT the problem is reduced to a set of self-consistent single particle equations, analogous to the Kohn-Sham equations for time-independent systems [5], with an effective potential of the form

$$
\begin{aligned}
v_{1}^{\text {eff }}(\vec{r}, \omega)= & v_{1}(\vec{r}, \omega)+\int \frac{n_{1}(\vec{r}, \omega)}{\left|\vec{r}-\vec{r}^{\prime}\right|} d \vec{r} \\
& +v_{1 \mathrm{xc}}(\vec{r}, \omega) ;
\end{aligned}
$$

the xc potential $v_{1 \mathrm{xc}}(\vec{r}, \omega)$ is linear in $n_{1}(\vec{r}, \omega)$,

$$
v_{1 \mathrm{xc}}(\vec{r}, \omega)=\int f_{\mathrm{xc}}\left(\vec{r}, \vec{r}^{\prime} ; \omega\right) n_{1}\left(\vec{r}^{\prime}, \omega\right) d \vec{r}^{\prime},
$$

and the kernel $f_{\mathrm{xc}}\left(\vec{r}, \vec{r}^{\prime} ; \omega\right)$ is a functional of the unperturbed ground state density $n_{0}(\vec{r})$.

In the spirit of the local density approximation Gross and Kohn (GK) [3] considered the case where both $n_{0}$ and $n_{1}$ are sufficiently slowly varying functions of $\vec{r}$. As $f_{\mathrm{xc}}$ is of short range for a homogeneous system, they proposed the following plausible approximation for systems of slowly varying $n_{0}(\vec{r})$ :

$$
f_{\mathrm{xc}}\left(\vec{r}, \vec{r}^{\prime} ; \omega\right) \sim f_{\mathrm{xc}}^{h}\left(\left|\vec{r}-\vec{r}^{\prime}\right|, \omega ; n_{0}(\vec{r})\right) .
$$

The superscript $h$ refers to a homogeneous electron gas and the function $f_{\mathrm{xc}}^{h}$ is a property of the homogeneous electron gas $[3,6]$.

However, it was noted later by Dobson [4] that the approximation (3), when applied to an electron gas in a static harmonic potential $\frac{1}{2} k r^{2}$ and subjected to a uniform electric field, $v_{1}(\vec{r}, \omega)=-\vec{E} \cdot \vec{r} e^{-i \omega t}$, violates the so-called harmonic potential theorem (HPT), related to the generalized Kohn's theorem [7], according to which the density follows rigidly the classical motion of the center of mass: $n_{1}(\vec{r}, \omega)=\vec{\nabla} n_{0}(\vec{r}) \cdot \vec{R}_{\mathrm{CM}}(\omega)$. This raised serious questions about the validity of the approximation (3). Dobson observed that one could satisfy the HPT by requiring that the GK approximation (3) be applied in a frame of reference moving with the local velocity of the electron fluid. The $\mathrm{xc}$ potential obtained by this construction is a functional of the current density as well as the density [8].

Further light on the problem with approximation (3) was thrown by Vignale's observation [9] that the covariance of the time-dependent Schrödinger equation under transformation to an accelerated frame of reference requires the total force exerted on the system by the exchange-correlation and Hartree potentials to vanish, in agreement with the third law of Newtonian mechanics. This implies that the exact $f_{\mathrm{xc}}$ must satisfy the sum rule [10]

$$
\int f_{\mathrm{xc}}\left(\vec{r}, \vec{r}^{\prime} ; \omega\right) \vec{\nabla}^{\prime} n_{0}\left(\vec{r}^{\prime}\right) d \vec{r}^{\prime}=\vec{\nabla} v_{0 \mathrm{xc}}(\vec{r}),
$$

where $v_{0 \mathrm{xc}}(\vec{r})$ is the static xc potential. This sum rule is violated by Eq. (3). More generally, one can deduce $[10,11]$ that $f_{\mathrm{xc}}\left(\vec{r}, \vec{r}^{\prime}, \omega\right)$ for a nonuniform system is of long range in space and a nonlocal functional of the density distribution. These results indicate that, contrary to more optimistic expectations, a local-density approximation for time-dependent linear response in general does 
not exist as long as one insists on describing dynamical exchange-correlation effects in terms of the density.

In this paper, we want to demonstrate, however, that a local approximation for the time-dependent linear response theory can be constructed in terms of the current density. We consider the linear current response $\vec{j}_{1}(\vec{r}, \omega) e^{-i \omega t}$ of interacting electrons in their ground state to a time-dependent vector potential $\vec{a}_{1}(\vec{r}, \omega) e^{-i \omega t}$. This problem includes, as a special case, the scalar potential problem studied by GK, because any scalar potential $v_{1}(\vec{r}, \omega)$ can be gauge transformed to a longitudinal vector potential $\vec{a}_{1}(\vec{r}, \omega)=\vec{\nabla} v_{1}(\vec{r}, \omega) / i \omega$, and the density response can be calculated from the current response using the continuity equation $n_{1}(\vec{r}, \omega)=\vec{\nabla} \cdot \vec{j}_{1}(\vec{r}, \omega) /$ $i \omega$. As usual, we express the exact induced current as the response of a noninteracting reference system (the "Kohn-Sham" system) to an effective vector potential $\vec{a}_{1}^{\text {eff }}=\vec{a}_{1}+\vec{a}_{1 H}+\vec{a}_{1 \mathrm{xc}}$,

$$
j_{1 i}(\vec{r}, \omega)=\int \sum_{j} \chi_{\mathrm{KS}, i j}\left(\vec{r}, \vec{r}^{\prime}, \omega\right) \cdot \vec{a}_{1 j}^{\mathrm{eff}}\left(\vec{r}^{\prime}, \omega\right) d \vec{r}^{\prime},
$$

where

$\vec{a}_{1 H}(\vec{r}, \omega)=\frac{1}{(i \omega)^{2}} \int \vec{\nabla} \frac{1}{\left|\vec{r}-\vec{r}^{\prime}\right|}\left[\vec{\nabla}^{\prime} \cdot \vec{j}_{1}\left(\vec{r}^{\prime}, \omega\right)\right] d \vec{r}^{\prime}$

is the longitudinal vector potential corresponding to the dynamic Hartree potential of Eq. (1), and

$$
\vec{a}_{1 \mathrm{xc}}(\vec{r}, \omega)=\int \mathbf{f}_{\mathrm{xc}}\left(\vec{r}, \vec{r}^{\prime}, \omega\right) \cdot \vec{j}_{1}\left(\vec{r}^{\prime}, \omega\right) d \vec{r}^{\prime}
$$

is a linear functional of the current. The $3 \times 3$ tensor kernel $\mathbf{f}_{x c}$ is defined as

$$
\begin{aligned}
\mathbf{f}_{x c, i j}\left(\vec{r}, \vec{r}^{\prime}, \omega\right)= & \chi_{\mathrm{KS}, i j}^{-1}\left(\vec{r}, \vec{r}^{\prime}, \omega\right)-\chi_{i j}^{-1}\left(\vec{r}, \vec{r}^{\prime}, \omega\right) \\
& -\nabla_{i} \frac{1}{\left|\vec{r}-\vec{r}^{\prime}\right|} \nabla_{j}^{\prime},
\end{aligned}
$$

where $\chi$ and $\chi_{\mathrm{KS}}$ are the current response tensors [12] of the interacting system and the "Kohn-Sham" noninteracting system, respectively. The latter is given as

$$
\begin{aligned}
\chi_{K S, i j}\left(\vec{r}, \vec{r}^{\prime}, \omega\right)= & \frac{n_{0}(\vec{r})}{m} \delta\left(\vec{r}-\vec{r}^{\prime}\right) \delta_{i j}+\frac{1}{m^{2}} \sum_{\alpha, \beta}\left(f_{\alpha}-f_{\beta}\right) \\
& \times \frac{\psi_{\alpha}^{*}(\vec{r}) \nabla_{i} \psi_{\beta}(\vec{r}) \psi_{\beta}^{*}\left(\vec{r}^{\prime}\right) \nabla_{j}^{\prime} \psi_{\alpha}\left(\vec{r}^{\prime}\right)}{\omega-\left(\epsilon_{\beta}-\epsilon_{\alpha}\right)+i \eta},
\end{aligned}
$$

where $\psi_{\alpha}(\vec{r})$ are the solutions of the static Kohn-Sham equation, with eigenvalues $\epsilon_{\alpha}$. (We have put $e=c=$ 1.) For a homogeneous electron gas of density $\bar{n}, \mathbf{f}_{\mathrm{xc}}$ is a function of $\vec{r}-\vec{r}^{\prime}$, which can be Fourier transformed to $\mathbf{f}_{\mathrm{xc}}^{h}(\vec{k}, \omega)$. In the limit of small wave vector $k$, which, at fixed frequency $\omega$, means $k \ll k_{F}, \omega / v_{F}\left(k_{F}\right.$ is the Fermi momentum and $v_{F}$ is the Fermi velocity) [13], $\mathbf{f}_{\mathrm{xc}}^{h}$ has the form

$$
\begin{aligned}
\mathbf{f}_{\mathrm{xc}, i j}^{h}(\vec{k}, \omega)=\frac{1}{\omega^{2}}[ & f_{\mathrm{xc} L}^{h}(\omega, \bar{n}) k_{i} k_{j}+f_{\mathrm{xc} T}^{h}(\omega, \bar{n}) \\
& \left.\times\left(k^{2} \delta_{i j}-k_{i} k_{j}\right)\right],
\end{aligned}
$$

where the factor $1 / \omega^{2}$ has been put in evidence so that the function $f_{\mathrm{xc} L}^{h}(\omega, \bar{n})$ coincides with the $k \rightarrow 0$ limit of $f_{\mathrm{xc}}^{h}(k, \omega, \bar{n})$ introduced by $G K$ [see Eq. (3)]. Both $f_{\mathrm{xc} L}^{h}(\omega, \bar{n})$ and $f_{\mathrm{xc} T}^{h}(\omega, \bar{n})$ can, in principle, be computed from the current response function of the homogeneous electron gas [see Eq. (8)]. While some aspects of these functions are known [3,14], further work is needed for a complete evaluation.

From Eq. (10) we can obtain the xc potential in real space for a homogeneous electron gas subjected to a perturbation which is slowly varying on the scales $k_{F}^{-1}$ ( $\sim$ interelectron distance) and $v_{F} / \omega(\sim$ distance traveled by an electron during a period of the perturbing field),

$$
\begin{aligned}
\vec{a}_{1 \mathrm{xc}}^{h}(\vec{r}, \omega)=\frac{1}{\omega^{2}}\left\{\vec{\nabla}\left[f_{\mathrm{xc} L}^{h}(\omega, \bar{n}) \vec{\nabla} \cdot \vec{j}_{1}(\vec{r}, \omega)\right]\right. \\
\left.-\vec{\nabla} \times\left[f_{\mathrm{xc} T}^{h}(\omega, \bar{n}) \vec{\nabla} \cdot \vec{j}_{1}(\vec{r}, \omega)\right]\right\} .
\end{aligned}
$$

Next, in the spirit of the local-density approximation, let us consider a system in which the unperturbed static density $n_{0}(\vec{r})$ is slowly varying on the scales of the local $k_{F}^{-1}$ and $v_{F} / \omega$, but not necessarily on the scale of $k^{-1}$, the wavelength of the perturbing field.

The simplest case is that of a periodically modulated electron gas, where the unperturbed density, given by

$$
n_{0}(\vec{r})=\bar{n}[1+2 \gamma \cos (\vec{q} \cdot \vec{r})],
$$

is not only slowly varying (i.e., $q \ll k_{F}, \omega / v_{F}$ ), but, also, almost uniform, i.e., $\gamma \ll 1$.

We shall compute the exact $\mathbf{f}_{\mathrm{xc}}$ for this system, to first order in $\gamma$. It is convenient to represent $\mathbf{f}_{\mathrm{xc}}$ in momentum space. Translational invariance of the uniform density $\bar{n}$ gives, to first order in $\gamma, \mathbf{f}_{\mathrm{xc}}(\vec{k}, \vec{k}, \omega)=\mathbf{f}_{\mathrm{xc}}^{h}(\vec{k}, \omega)$ [see Eq. (10)] and $\mathbf{f}_{\mathrm{xc}}(\vec{k}+m \vec{q}, \vec{k}, \omega)=0$ for integers $m$ with $|m|>1$. It remains to calculate the matrix element $\mathbf{f}_{\mathrm{xc}}(\vec{k}+\vec{q}, \vec{k}, \omega)$.

This is facilitated by two exact identities which follow from the transformation of the time-dependent Schrödinger equation in accelerated reference frames [15]. The physical content of these identities is that the total force and the total torque exerted by the Hartree and xc potentials on the system must vanish, in accordance with Newton's third law. In the specific instance considered here, the identities take the form

$$
\begin{aligned}
& \operatorname{limf}_{k \rightarrow 0} \mathbf{f c}, i j_{1}(\vec{k}+\vec{q}, \vec{k}, \omega) \\
& \quad=-\frac{\gamma}{\omega^{2}}\left[\delta f_{\mathrm{xc} L}^{h} q_{i} q_{j}+f_{\mathrm{xc} T}^{h}\left(q^{2} \delta_{i j}-q_{i} q_{j}\right)\right]
\end{aligned}
$$


and

$$
\begin{aligned}
\lim _{k \rightarrow 0} \sum_{j, k} \epsilon_{l j k} \frac{\partial}{\partial k_{k}} \mathbf{f}_{\mathrm{xc}, i j}(\vec{k}+\vec{q}, \vec{k}, \omega) \\
=-\frac{\gamma}{\omega^{2}}\left[\delta f_{\mathrm{xc} L}^{h}-3 f_{\mathrm{xc} T}^{h}\right] \sum_{k} \epsilon_{l k i} q_{k},
\end{aligned}
$$

where $\epsilon_{i j k}$ is the Levi-Civita tensor and we have suppressed $\omega$ and $\bar{n}$ in $f_{\mathrm{xc} L}^{h}$ and $f_{\mathrm{xc} T}^{h}$; and

$$
\delta f_{\mathrm{xc} L}^{h}(\omega, \bar{n}) \equiv f_{\mathrm{xc} L}^{h}(\omega, \bar{n})-f_{\mathrm{xc} L}^{h}(\omega=0, \bar{n}) .
$$

These identities are valid for $k, q \ll k_{F}, \omega / v_{F}$ and to first order in $\gamma$. The condition on $q$ has been used to replace $f_{\mathrm{xcL} L}^{h}(q, \omega)$ and $f_{\mathrm{xc} T}^{h}(q, \omega)$ by their small $q$ limit [13]. A third limiting form of $\mathbf{f}_{\mathrm{xc}, i j}$ is obtained from the usual
Ward identity [12]

$$
\lim _{q \rightarrow 0} \mathbf{f}_{\mathrm{xc}}(\vec{k}+\vec{q}, \vec{k}, \omega)=\gamma \bar{n} \frac{\partial \mathbf{f}_{\mathrm{xc}}^{h}(\vec{k}, \omega, \bar{n})}{\partial \bar{n}} .
$$

Finally, we note the symmetry relation

$$
\mathbf{f}_{\mathrm{xc}, i j}(\vec{k}+\vec{q}, \vec{k}, \omega)=\mathbf{f}_{\mathrm{xc}, j i}(-\vec{k},-\vec{k}-\vec{q}, \omega),
$$

which holds for a system invariant under time reversal. Equations (13)-(17) require that, for small $k$ and $q$, $\mathbf{f}_{\mathrm{xc}, i j}(\vec{k}+\vec{q}, \vec{k}, \omega)$ be expressible as a linear combination of $q_{i} q_{j}, q^{2} \delta_{i j},\left(k_{i}+q_{i}\right) k_{j}, k_{i}\left(k_{j}+q_{j}\right)$, and $\vec{k}$. $(\vec{k}+\vec{q}) \delta_{i j}$. Equations (13), (14), and (16), completely determine the coefficients of this combination. Thus, to first order in $\gamma$, we obtain

$$
\begin{gathered}
\mathbf{f}_{\mathrm{xc}, i j}(\vec{k}+\vec{q}, \vec{k}, \omega)=-\frac{\gamma}{\omega^{2}}\left\{\left(\delta f_{\mathrm{xc} L}^{h}-f_{\mathrm{xc} T}^{h}\right) q_{i} q_{j}+f_{\mathrm{xc} T}^{h} q^{2} \delta_{i j}-\bar{n} \frac{\partial f_{\mathrm{xc} T}^{h} \vec{k} \cdot(\vec{k}+\vec{q}) \delta_{i j}+A(\bar{n}, \omega)}{\partial \bar{n}}\right. \\
\left.\times\left(k_{i}+q_{i}\right) k_{j}-B(\bar{n}, \omega) k_{i}\left(k_{j}+q_{j}\right)\right\},
\end{gathered}
$$

where $A(\bar{n}, \omega) \equiv\left[\bar{n}\left(2 \partial f_{\mathrm{xc} T}^{h} / \partial \bar{n}-\partial f_{\mathrm{xc} L}^{h} / \partial \bar{n}\right)+3 f_{\mathrm{xc} T}^{h}-\right.$ $\left.\delta f_{\mathrm{xc} L}^{h}\right]$ and $B(\bar{n}, \omega) \equiv\left[\bar{n} \partial f_{\mathrm{xc} T}^{h} / \partial \bar{n}+3 f_{\mathrm{xc} T}^{h}-\delta f_{\mathrm{xc} L}^{h}\right]$. The essential point is that Eq. (18) is analytic for small $k$ and $q$. It is this feature that will enable us to construct a local approximation for $\vec{a}_{1 \mathrm{xc}}$ in terms of the current density. [By contrast, the off-diagonal component $f_{\mathrm{xc}}(\vec{k}+\vec{q}, \vec{k}, \omega)$ in the usual time-dependent density functional theory has a singularity of the form $\vec{k} \cdot \vec{q} / k^{2}$ when $k \rightarrow 0$ at finite $q$. This small- $k$ singularity reflects the already mentioned long rangedness of $f_{\mathrm{xc}}$ and the consequent nonlocality of the description of xc effects in terms of the density.]

Let us now return to the more general problem of determining $\vec{a}_{1 \mathrm{xc}}$ for a system whose density is slowly varying in the sense $\left|\vec{\nabla} n_{0}(\vec{r})\right| / n_{0}(\vec{r}) \ll k_{F}(\vec{r})$ and $\omega / v_{F}(\vec{r})$, but may have large global deviations from uniformity. We assume that $\vec{a}_{1 \times c}$ of such a system can be expanded in a power series of gradients of the local unperturbed density. The most general form of $\vec{a}_{1 \mathrm{xc}}$ containing up to two gradient operators is then a linear combination of the following terms: $\vec{j}, \vec{\nabla} n_{0} \times \vec{j}_{1}$, $\vec{\nabla} \times \vec{j}_{1}, \quad \vec{\nabla}\left(\vec{\nabla} \cdot \overrightarrow{j_{1}}\right), \quad \vec{\nabla} \times\left(\vec{\nabla} \times \vec{j}_{1}\right), \vec{\nabla}\left(\vec{\nabla} n_{0} \cdot \overrightarrow{j_{1}}\right), \vec{\nabla} \times$ $\left(\vec{\nabla} n_{0} \times \overrightarrow{j_{1}}\right), \vec{\nabla} n_{0}\left(\vec{\nabla} \cdot \overrightarrow{j_{1}}\right), \vec{\nabla} n_{0} \times\left(\vec{\nabla} \times \overrightarrow{j_{1}}\right),\left(\vec{\nabla} n_{0} \cdot \vec{\nabla}\right) \overrightarrow{j_{1}}$, $\vec{\nabla} n_{0}\left(\vec{\nabla} n_{0} \cdot \vec{j}_{1}\right), \vec{\nabla} n_{0} \times\left(\vec{\nabla} n_{0} \times \vec{j}_{1}\right)$ with coefficients that are functions of $n_{0}(\vec{r})$ and $\omega$. This gradient expansion is applicable in particular, to the periodically modulated electron gas of Eq. (12), in which case it must yield the same perturbative (in $\gamma$ ) result that one obtains from the combination of Eqs. (7), (10), and (18). Thus, by requiring agreement between the gradient and the perturbative expansions, we are able to determine the coefficients of all the terms appearing in the gradient expansion, except the last two, which are of order $\left|\vec{\nabla} n_{0}\right|^{2}$, or $\gamma^{2}$ in the periodically modulated electron gas. We note that the part of $\vec{a}_{1 \mathrm{xc}}$ which is determined by this procedure satisfies the HPT up to corrections of order $\left|\vec{\nabla} n_{0}\right|^{2}$. To fix the coefficients of the second order terms, an additional condition must be imposed. We require that if the system is subjected to an external field, which causes it to translate rigidly as a whole, i.e., $j_{1}(\vec{r}, \omega)=n_{0}(\vec{r}) \vec{v}(\omega)$, then $\vec{a}_{1 \mathrm{xc}}$ must reduce to $-\vec{\nabla}\left[f_{\mathrm{xc} L}(\omega=0) \vec{\nabla} \cdot j_{1}(\vec{r}, \omega)\right] / \omega^{2}$, a rigid displacement of the static exchange-correlation potential [9]. In the special case of electrons confined by a harmonic potential and subjected to a uniform electric field, this condition guarantees that the HPT is satisfied.

The final result of our analysis, obtained after lengthy but elementary manipulations, is the complete form of the local approximation for $\vec{a}_{1 \times c}(\vec{r}, \omega)$ up to second order in the gradient expansion,

$$
\begin{aligned}
& \vec{a}_{1 \mathrm{xc}}(\vec{r}, \omega)=-\frac{1}{\omega^{2}}\left\{\vec{\nabla}\left[f_{\mathrm{xcL}}^{h} \vec{\nabla} \cdot \vec{j}_{1}(\vec{r}, \omega)-\delta f_{\mathrm{xc} L}^{h} \vec{\nabla} n_{0}(\vec{r}) \cdot \frac{\vec{j}_{1}(\vec{r}, \omega)}{n_{0}(\vec{r})}\right]-\vec{\nabla} \times\left[f_{\mathrm{xc} T}^{h} n_{0} \vec{\nabla} \times \frac{\vec{j}_{1}(\vec{r}, \omega)}{n_{0}(\vec{r})}\right]\right.
\end{aligned}
$$

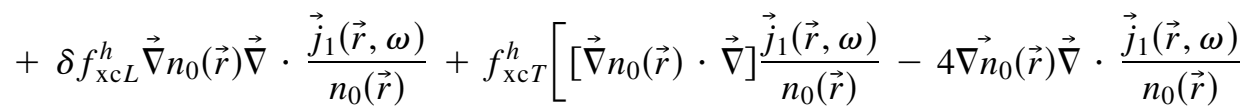

$$
\begin{aligned}
& \left.\left.+3 \sum_{j} \nabla_{j} n_{0}(\vec{r}) \vec{\nabla} \frac{\vec{j}_{1 j}(\vec{r}, \omega)}{n_{0}(\vec{r})}\right]+2 n_{0}(\vec{r})\left[\sum_{j} \nabla_{j} f_{\mathrm{xc} T}^{h} \vec{\nabla} \frac{\vec{j}_{1}(\vec{r}, \omega)}{n_{0}(\vec{r})}-\vec{\nabla} f_{\mathrm{xc} T}^{h} \vec{\nabla} \cdot \frac{\vec{j}_{1}(\vec{r}, \omega)}{n_{0}(\vec{r})}\right]\right\},
\end{aligned}
$$

where $f_{\mathrm{xcL} L}^{h}$ and $f_{\mathrm{xc} T}^{h}$ are functions of the local density $n_{0}(\vec{r})$ and the frequency $\omega$. 
Our result simplifies considerably in two limiting cases. (1) The density is slowly varying on the scale set by the wavelength of the perturbation. In this regime we can neglect the terms containing $\vec{\nabla} n_{0}$, and we recover the former results of GK [1] for the response to a scalar potential, and of $\mathrm{Ng}$ [16] for the response to a general vector potential. This result can also be simply obtained from Eq. (11) by the substitution $\bar{n} \rightarrow n_{0}(\vec{r})$. (2) The velocity field $j_{1} / n_{0}$ is constant in space. This is the case when the static external potential is parabolic and the perturbing electric field is uniform, the regime of Kohn's theorem [7] and the HPT [4]. Then all derivatives of $j_{1} / n_{0}$ vanish, and Eq. (19) reduces to

$$
\begin{aligned}
\vec{a}_{1 \mathrm{xc}}(\vec{r}, \omega)=-\frac{1}{\omega^{2}} \vec{\nabla}[ & f_{\mathrm{xc} L}^{h} \vec{\nabla} \cdot \vec{j}_{1}(\vec{r}, \omega) \\
& \left.\quad-\delta f_{\mathrm{xc} L}^{h} \vec{\nabla} n_{0}(\vec{r}) \cdot \frac{\vec{j}_{1}(\vec{r}, \omega)}{n_{0}(\vec{r})}\right] .
\end{aligned}
$$

By a gauge transformation, this longitudinal vector potential can be transformed to the scalar xc potential proposed by Dobson [4] to satisfy the HPT. However, for the general case of slowly varying $n_{0}(\vec{r})$ and $\vec{a}_{1}(\vec{r}, \omega)$, the full expression (19) is required.

In conclusion, our analysis uniquely specifies a local current density functional theory of the linear current (and density) response, which becomes exact in the limit of slowly varying unperturbed densities and perturbing potentials. The scale on which the variations must be slow is set by the smaller of the wave vectors $k_{F}$ and $\omega / v_{F}$. Therefore, this theory is applicable to the study of high frequency phenomena, such as electromagnetic absorption, for which the adiabatic approximation [2] is in general not justified. A complete local current density response theory, for spatially slowly varying unperturbed density and perturbing field, and all frequencies $(0<\omega<\infty)$ remains to be developed.

We acknowledge support from NSF Grants No. DMR9403908 and No. DMR-9308011. One of us (G. V.) gratefully acknowledges the hospitality of the Institute of Theoretical Physics and of the Physics Department at
UCSB, where this work was initiated. We thank A. K. Rajagopal for several stimulating discussions.

[1] E. Runge and E. K. U. Gross, Phys. Rev. Lett. 52, 997 (1984).

[2] A. Zangwill and P. Soven, Phys. Rev. Lett. 45, 204 (1980); Phys. Rev. B 24, 4121 (1981).

[3] E. K. U. Gross and W. Kohn, Phys. Rev. Lett. 55, 2850 (1985). See also E. K. U. Gross and W. Kohn, Adv. Quantum Chem. 21, 255 (1990).

[4] J. Dobson, Phys. Rev. Lett. 73, 2244 (1994). See also Density Functional Theory, edited by E. K. U. Gross and R. M. Dreizler, NATO ASI (Plenum, New York, 1994), p. 393.

[5] W. Kohn and L. Sham, Phys. Rev. 140, A1133 (1965).

[6] H. M. Böhm, S. Conti, and M.P. Tosi, J. Phys. Condens. Matter 8, 781 (1996).

[7] W. Kohn, Phys. Rev. 123, 1242 (1961); L. Brey et al., Phys. Rev. B 40, 10647 (1989); 42, 1240 (1990); S. K. Yip, Phys. Rev. B 43, 1707 (1991).

[8] This construction has recently been extended to the nonlinear regime; see M. Bünner, J. Dobson, and E. K. U. Gross (to be published).

[9] G. Vignale, Phys. Rev. Lett. 74, 3233 (1995).

[10] G. Vignale, Phys. Lett. A 209, 206 (1995).

[11] G. Vignale and W. Kohn (unpublished).

[12] P. Noziéres, The Theory of Interacting Fermi Systems (W. A. Benjamin, New York, 1964), Chap. 6.

[13] The response functions of the homogeneous electron gas change behavior as $k$ crosses over from $k<\omega / v_{F}$ to $k>\omega / v_{F}$. Our "small $k$ " limit refers to the first regime.

[14] We have obtained a few exact results about the function $f_{\mathrm{xc} T}^{h}(\omega, n)$ similar to the ones already known for $f_{\mathrm{xc} L}^{h}(\omega, n)$. To first order in the Coulomb interaction, $f_{\mathrm{xc} T}^{h}(\omega, n)$ is independent of frequency and equal to $3 \pi e^{2} / 10 k_{F}^{2}$. The limit of $f_{\mathrm{xc} T}^{h}$ for $\omega \rightarrow \infty$ is given by $\frac{2}{5} n^{2 / 3}(d / d n)\left[\epsilon_{\mathrm{xc}}(n) / n^{2 / 3}\right]+2 n^{1 / 3}(d / d n)\left[\epsilon_{\mathrm{xc}}(n) / n^{1 / 3}\right]$ where $\epsilon_{\mathrm{xc}}(n)$ is the exchange-correlation energy per particle of the uniform electron gas of density $n$.

[15] A detailed derivation and discussion of these "acceleration identities" will be published elsewhere.

[16] Tai Kai Ng, Phys. Rev. Lett. 62, 2417 (1989). 